\title{
Incidence, Etiopathogenesis, and a Preventive Protocol of Deep Vein Thrombosis in Severe Burns Patients at a Tertiary Care Teaching Hospital
}

\author{
R Ravishanker ${ }^{1}$, Danturti Niharika ${ }^{2}$
}

\begin{abstract}
Deep vein thrombosis (DVT) a consequence of thromboembolism can lead to life threatening pulmonary embolism. A study was carried over 4 years from January 2014 to January 2018 to determine the incidence of DVT in severe burns cases admitted to the Burns Unit of a 1200 bed tertiary care medical college teaching hospital. In the retrospective study of 100 consecutive cases of severe burns the incidence of DVT was found to inordinately high. This study was carried out to determine the causes of DVT in these patients and formulate a protocol for the prevention of DVT. A further prospective study of 116 cases were studied following the introduction of the protocol. A dramatic decrease in the incidence was found. This protocol is recommended to be followed in all burns centres especially in teaching institutions.

Keywords: Deep vein thrombosis, High incidence, Protocol for prevention, Severe burns patients, Teaching institution.

Journal of Medical Academics (2019): 10.5005/jp-journals-10070-0030
\end{abstract}

\section{INTRODUCTION}

Deep vein thrombosis (DVT) is a multifactorial condition that develops in the deep veins of the lower limb. Venous thromboembolism (VTE) is a general term associated with DVT; its severe fatal consequence is pulmonary embolism (PE). ${ }^{1-4}$ DVT is a semisolid clot formed usually in the deeper vein of the leg that under severe cases detaches and travels to the lungs to cause acute pulmonary inflammation., 5 ainful edema and congestion in the leg occurs owing to the occlusion of the femoral vein. Extensive DVT of iliac and pelvic veins cause phlegmasia, in which either venous gangrene of infarction may threaten a part or the whole limb. ${ }^{3-6}$

A recorded occurrence of DVT in the western world is one per 1,000 population per year in the hospitalized general population. ${ }^{6-8}$ The incidence in India is higher in men $(114 / 10,00,000)$ than in women $(105 / 1,00,000) .{ }^{2}$ Approximately, one-third of the patients with symptomatic VTE manifest PE; two-thirds of the patients manifest DVT. ${ }^{6-8}$

Statistics of burn patients with DVT are scarce owing to a dearth of literature and records available in this demographic setting. Though western literature has some works reporting an incidence of $0.25-23 \%$, the studies are limited. ${ }^{7-11}$ There are practically no studies available for the same in India.

Severe burn patients are at a higher risk of manifesting DVT owing to the intensity of burns and longer periods of hospitalization and immobilization. Some of the sequelae include death due to pulmonary edema and gangrene of the affected part. 3,7,9,10

Diagnosis is often missed in these cases. Diagnosis involves a combination of clinical awareness and examination combined with a Duplex scan for confirmation of DVT. ${ }^{10-13}$

Prophylaxis in the form of low molecular weight heparin (LMWH) has been advised to prevent DVT in critical cases. ${ }^{4,710,13}$ However, the problem of administering LMWH in burn patients is the extensive raw areas, which can lead to bleeding and severe anemia. It is not easy for heparinized patients and patients on anticoagulants with extensive burns to be taken up for surgery because of heavy bleeding from raw areas and donor site during
${ }^{1}$ Burns and Reconstructive Surgery Unit, Pravara Rural Hospital of the Rural Medical College, Pravara Institute of Medical Sciences, Loni, Maharashtra, India

${ }^{2}$ Rural Medical College, Pravara Institute of Medical Sciences, Loni, Maharashtra, India

Corresponding Author: R Ravishanker, Burns and Reconstructive Surgery Unit, Pravara Rural Hospital of the Rural Medical College, Pravara Institute of Medical Sciences, Loni, Maharashtra, India, e mail: reconsravi@gmail.com

How to cite this article: Ravishanker R, Niharika D. Incidence, Etiopathogenesis, and a Preventive Protocol of Deep Vein Thrombosis in Severe Burns Patients at a Tertiary Care Teaching Hospital. J Med Acad 2019;2(1):6-10.

Source of support: Nil

Conflict of interest: None

surgery and this has prevented a lot of physicians treating burns from initiating prophylaxis for DVT. ${ }^{14}$

In view of the aforementioned sequelae of DVT and its complications, it was thought prudent to evaluate patients of burns presenting with DVT in this 18 bed burns unit of a 1,200 bed tertiary care hospital with an average admission of about 200 severe burns patients annually.

\section{Materials and Methods}

This was a retrospective and prospective study from January 2014 to January 2018 in the burns unit of the Pravara Rural Hospital of the Pravara Institute of Medical Sciences, Loni, Maharashtra. The study was carried out after obtaining Institutional Ethics Clearance (IEC).

Enrolled patients were subjected to the following inclusion and exclusion criteria.

\section{Inclusion Criteria}

- Adult patients (age ranging from 1868) admitted with severe burns from January 2014 and diagnosed with DVT.

() The Author(s). 2019 Open Access This article is distributed under the terms of the Creative Commons Attribution 4.0 International License (https://creativecommons. org/licenses/by-nc/4.0/), which permits unrestricted use, distribution, and non-commercial reproduction in any medium, provided you give appropriate credit to the original author(s) and the source, provide a link to the Creative Commons license, and indicate if changes were made. The Creative Commons Public Domain Dedication waiver (http://creativecommons.org/publicdomain/zero/1.0/) applies to the data made available in this article, unless otherwise stated. 
- Severe burns was a burn surface area of $20 \%$ and over.

- Patients of either gender.

- Patients with burns ready to give written informed consent.

- All hospitalized burns patients with DVT and ready to stay till considered fit for discharge.

\section{Exclusion Criteria}

- Pediatric-age-group patients.

- Patients with burns not willing to stay or be hospitalized for the entire duration of the treatment.

- Records of patients that were incomplete for retrospective study.

- Patients with burns with a prior history of ischemic heart disease, DVT, hypercoagulable states, malignancy, frequent blood transfusions, and absence of coagulation factors.

- Study conduct-all patients satisfying the above inclusion and exclusion criteria were subjected to the following variables.

\section{Demographic Variables}

- Age, gender

- Socioeconomic status

- Rural/urban area

- Educational status

- Body weight

\section{History of Burns}

- Degree of burns, total surface area, area involved

\section{DVT Parameters}

- Occurrence

- Location of thrombus

- Clinical presentation

- Complication

- Treatment protocol

\section{Outcome of DVT}

- Morbidity

- Mortality

- Status at discharge

\section{Sample Size}

100 retrospective cases and 116 prospective cases

\section{Data Analysis}

All the data related to above variables that were recovered from the case records forms tabulated in an excel sheet. Observation tables were prepared according to objectives to be satisfied and subjected to statistical tests.

\section{Statistical Analysis}

Descriptive statistics used were percentage mean, median and mode, inferential statistics, test of significance appropriate observation to draw inference.

The retrospective study was from the period January 2014July 2015. The prospective study started from July 2015 after the initiation of a protocol for thromboprophylaxis of DVT by both mechanical and drug-based methods.

\section{Observation and Results}

\section{Retrospective Study}

A sample size of a 100 burn patients for the retrospective study was considered from the period starting from January 2014, and the incidence of DVT amongst them along with the correlation of each risk factor was measured. The incidence of DVT amongst burns patients was found to be 10 out of 100 , amounting to $10 \%$ of the total study size (Table 1).

The age group considered was 18-68 for the study. Of the 34 patients (age: 18-28 years) suffered from burns, DVT was seen in 1 patient. In 37 patients ranging from the age group 29-38, 9 patients with DVT out of this study size was found. No DVT was found in any of the other age groups (Table 2).

In the gender-wise occurrence of DVT amongst the 10 affected people, 4 were female, amounting to a $40 \%$ occurrence and 6 were males, amounting to $60 \%$. It can be observed that the incidence was almost the same in both females and males about 10\% (Table 3).

The percentage of burns and its correlation to the occurrence of DVT was assessed. The percentage range was divided into a section of ten. No patients of DVT were seen in the percentages of burns ranging from 0 to $10 \%$ and from 10 to $20 \%$. In the burns percentage, in the range of $20-30 \%$, there was one patient with DVT. In the range of $30-40 \%$, there were 3 patients with DVT. In the range of $40-50 \%$ and $50-60 \%$, there were 2 patients each. Only 1

Table 1: Incidence of DVT; retrospective study

\begin{tabular}{lll}
\hline Number of patients & $\begin{array}{l}\text { Number of patients } \\
\text { with DVT }\end{array}$ & Incidence \\
\hline 100 & 10 & $10 \%$ \\
\hline
\end{tabular}

Table 2: Age-wise distribution of patients with DVT; retrospective study

\begin{tabular}{lll}
\hline Age group & $\begin{array}{l}\text { Number of burns } \\
\text { patients }\end{array}$ & Patients with DVT \\
\hline $18-28$ & 34 & 1 \\
$29-38$ & 37 & 9 \\
$39-48$ & 13 & 0 \\
$49-58$ & 9 & 0 \\
$59-68$ & 7 & 0 \\
\hline
\end{tabular}

Table 3: Gender distribution of patients with DVT; retrospective study

\begin{tabular}{|c|c|c|c|}
\hline Gender & $\begin{array}{l}\text { Number of } \\
\text { patients }\end{array}$ & $\begin{array}{l}\text { Number of } \\
\text { patients with } \\
\text { DVT }\end{array}$ & Incidence (\%) \\
\hline Female & 43 & 4 & 9.4 \\
\hline Male & 57 & 6 & 10.6 \\
\hline
\end{tabular}

Table 4: Burns percentage with DVT distribution; retrospective study

\begin{tabular}{ll}
\hline Percentage of burns (\%) & Number of patients with DVT \\
\hline $0-10$ & 0 \\
$11-20$ & 0 \\
$21-30$ & 1 \\
$31-40$ & 3 \\
$41-50$ & 2 \\
$51-60$ & 2 \\
$61-70$ & 1 \\
Electric burns & 1 \\
\hline
\end{tabular}


patient was found to have DVT in the range of $60-70 \%$ burns. The one patient with electric burns and DVT had a high-tension burn injury (Table 4).

Of the 10 patients with DVT, 8 were smokers, with 2 of them being women. Only 2 of $10(20 \%)$ DVT patients were intubated for inhalational injuries. Intubation is known to be a factor for causation of DVT.

With the difference in time between the incidence of thermal injury and admission to PRH, Loni was found to be significant and relevant. An estimated 72 hours is the border for the setting of risk factors. Beyond 72 hours or 3 days is indicative of significance as a risk factor. Of the 10 (70\%) patients, 7 were admitted within 72 hours of having sustained burns. Only 3 of $10(30 \%)$ patients had beyond a 72-hours delay in admission (Table 5).

Femoral lines were being inserted when the percentage of burns are beyond $20 \%$. It was found that all the 10 patients of DVT (100\%) had femoral lines inserted in the casualty prior to admission into the ward, which is a prominent risk.

\section{Prospective Study}

The prospective study was started in July 2015 after a protocol was initiated for the prevention and management of DVT.

The incidence of DVT markedly dropped after the adoption of the new protocol. The only case was a 26 year female with about $56 \%$ thermal burns admitted on the fourth day after having sustained burns. She had been treated in another hospital and came with a femoral line in the affected limb.

Table 5: Time gap between incidence of burns and DVT; retrospective study

\begin{tabular}{ll}
\hline Less than 72 hours & More than 72 hours \\
\hline 7 & 3 \\
\hline
\end{tabular}

Table 6: Age-wise distribution of patients with DVT; prospective study

\begin{tabular}{lll}
\hline Age group & Number of patients & Patients with DVT \\
\hline $18-28$ & 39 & $\begin{array}{l}1 \text { (patient admitted with } \\
\text { DVT) }\end{array}$ \\
$29-38$ & 41 & 0 \\
$39-48$ & 16 & 0 \\
$49-58$ & 13 & 0 \\
$59-68$ & 7 & 0 \\
\hline
\end{tabular}

Table 7: Gender distribution of patients with DVT; prospective study

\begin{tabular}{llll}
\hline & $\begin{array}{l}\text { Number of } \\
\text { patients }\end{array}$ & $\begin{array}{l}\text { Number of } \\
\text { patients with } \\
\text { DVT }\end{array}$ & Incidence (\%) \\
\hline Fender & 57 & 1 & 1.75 \\
Male & 65 & 0 & 0 \\
\hline
\end{tabular}

Table 8: Burns percentage with DVT distribution; prospective study

\begin{tabular}{ll}
\hline Percentage of burns (\%) & Number of patients with DVT \\
\hline $0-10$ & 0 \\
$11-20$ & 0 \\
$21-30$ & 0 \\
$31-40$ & 0 \\
$41-50$ & 1 \\
$51-60$ & 0 \\
$61-70$ & 0 \\
Electric burns & 0 \\
\hline
\end{tabular}

Parameters with reference to the age, sex, and percentage of burns are represented in the following tables (Tables 6 to 8).

\section{Discussion}

Deep vein thrombosis is a consequence of venous thromboembolism, which on progression in certain cases leads to a life-threatening condition called pulmonary embolism. ${ }^{1,2,5,10}$ Deep vein thrombosis is a debilitating condition with an increase in worldwide occurrence found in various studies. $2,7,8,11,13$

A number of factors predispose a patient to DVT, with the predominant factor being Virchow's triad, which comprises three aspects: hypercoagulability, stasis of blood, injury to vessel wall, or endothelial injury. Tobacco, oral contraceptives, cigarette smoking, HRT, and obesity are considerable causative risk factors for the disease. ${ }^{11,13,15,16}$

Diagnosis starts with history (risk factors) and physical examination using a validated clinical prediction rule, such as the Wells DVT score. ${ }^{5,6,8,9}$ Patients with a high likelihood of DVT are tested with duplex compression ultrasonography, where the length of the proximal veins is sequentially compressed with an ultrasound probe. ${ }^{5,6,17}$ Normal veins are easily occluded with moderate external compression, but a DVT will prevent occlusion of the vein lumen. USG is both sensitive and specific for DVTs. ${ }^{17}$ Contrast venography is considered the gold standard for diagnosis of DVT, although rarely done owing to its invasiveness, expense, availability, and risks involved. $5,6,9$

Prophylaxis should be started from admission. $3,5,6,15,18$

Prophylaxis includes the use of foot or leg sequential compression devices in every patient. Pneumatic compression devices have been shown to reduce thromboembolic events by more than half without increasing bleeding risk by preventing venous pooling and activating fibrinolysis. ${ }^{19}$ In patients with higher risk factors such as extensive burns, genetic thrombophilia, and history of prior deep venous thromboembolism or pulmonary embolism, low molecular weight heparin (LMWH) is given. LMWH appears to be at least as effective as standard heparin in DVT prophylaxis with possibly less bleeding tendencies. ${ }^{17,18,20-23}$

Treatment of DVT is in the form of pharmacological intervention assisted by mechanical intervention. ${ }^{18,21,23}$ Pharmacological intervention uses drugs like LMWH and warfarin as parenteral and oral anticoagulants respectively, while mechanical intervention includes the use of compression stockings. Blood thinners such as Fondaparinux and promoters of coagulation factors such as vitamin $\mathrm{K}$ are also to be given along with a protein-rich diet. ${ }^{18,21,23}$

\section{Purpose of this Study}

This study was conducted in the hope of providing adequate information on the incidence and etiopathology of DVT in cases of burns, as there is not much literature available on this subject. ${ }^{7-11}$ No study has been performed on DVT in burns patients in India to use as a template and corroborate as evidence or contradict in terms of the results obtained, though a study has been done on the role of thrombopropylaxis with low-molecular-weight heparin. ${ }^{14}$

The reason that this study was taken up was on account of an alarming incidence of DVT in the burns unit. The observations put forward are the results of a thorough study performed with the resources and the study size available at Pravara Rural Hospital, Loni in the burns unit.

It was found that the percentage of DVT patients in the sample size of 100 was $10 \%$, which is high considering the global occurrence percentage of $0.25-23 \% .^{7-11}$ 
The incidence of DVT was marginally higher in males when compared to females and this could be attributed to tobacco consumption. Tobacco consumption was found in a high percentage of $80 \%$ amongst the DVT patients; all of the men and two women were users of tobacco.

Oral contraceptives or HRT (another risk factor for DVT) was not seen as a positive finding in any of the female patients. Patients in rural areas consider oral contraceptives to be taboo and do not undergo any such reproductive interventions.

Obesity is another common risk factor for DVT as it may lead to deposition of cholesterol in the lumen of the vessels (possibly atherosclerosis), which again causes endothelial damage. None of the patients were found to be obese as obesity is a common finding among the affluent societies, which is not again usually found in rural areas.

An estimated $20 \%$ of the patients with DVT were intubated and on ventilator support, hence were totally immobile, predisposing to stasis in the lower limbs.

Only $30 \%$ patients were admitted 72 hours after the thermal injury, which is enough time for hypercoagulability to take place if adequate fluid resuscitation had not been done.

A very important finding in the study was that there was a tradition in the hospital of insertion of femoral lines for patients with burns over $20 \%$. All 10 of 10 patients with DVT had femoral lines

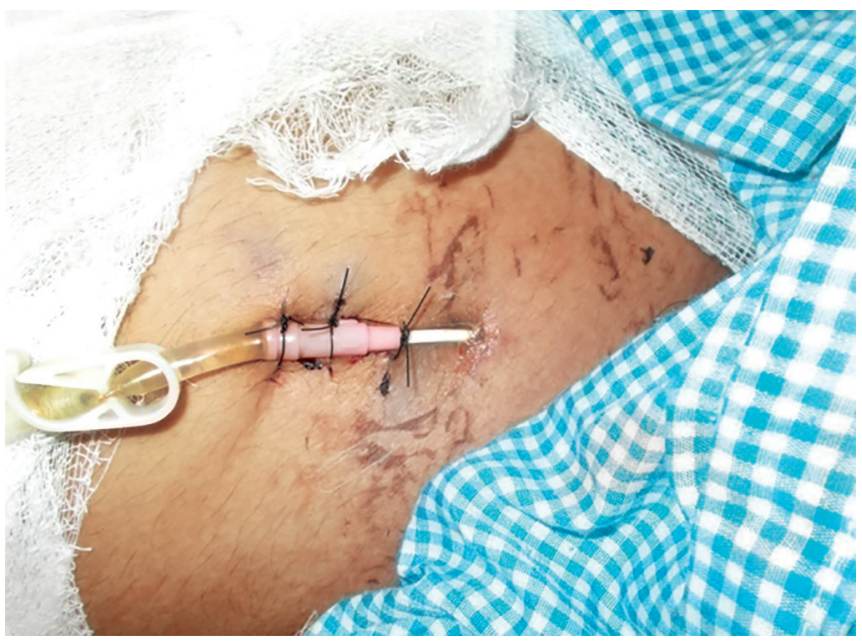

Fig. 1: Exposed cannula femoral region

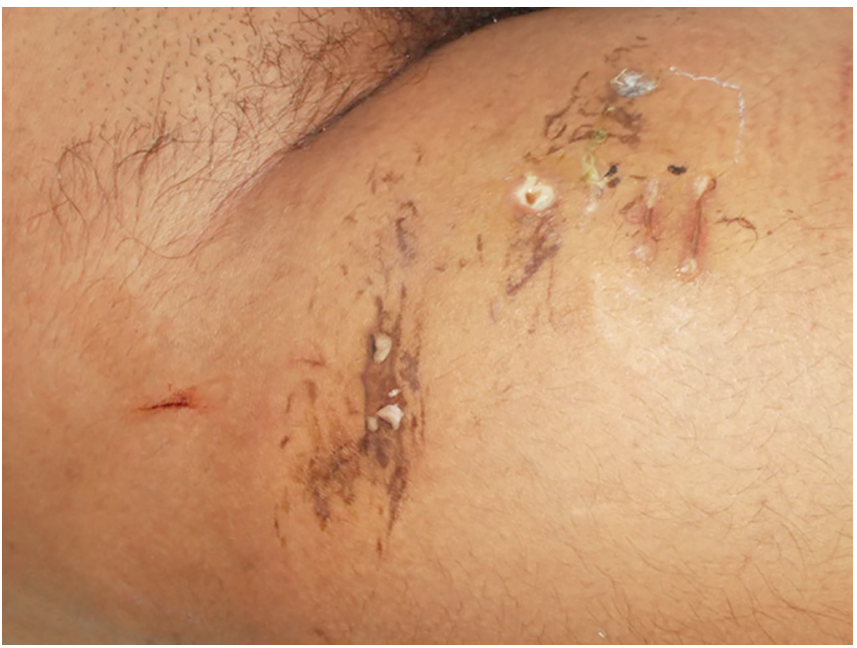

Fig. 3: Multiple puncture attempts inserted. It was found that the femoral lines, albeit inserted on time, were not inserted in the right manner or were either inserted after multiple attempts (Figs 1 to 4). This caused injury to major vessel walls, which then brought the Virchow's triad into play. A further investigation revealed that the root cause of this was the insertion of these lines performed in the casualty by the junior-most and unsupervised surgical residents.

There also was no practice of patients being placed on prophylactic therapy for the prevention of DVT. It was feared that in view of extensive raw areas in burn wounds, prophylactic treatment for DVT would increase bleeding during dressings.

There was no protocol as such for prevention of or detection of DVT.

Clinically all patients with confirmed DVT had shown the manifestations of DVT in terms of leg swelling, edema, gangrene, and pain in the lower limbs. Homan's and Moses' sign were positive. Vascular Doppler studies were performed on all the patients to confirm the DVT and were positive in all cases.

\section{Protocols Initiated}

No femoral line insertion was to be done in the casualty/emergency room. If considered necessary to be done by an experienced faculty.

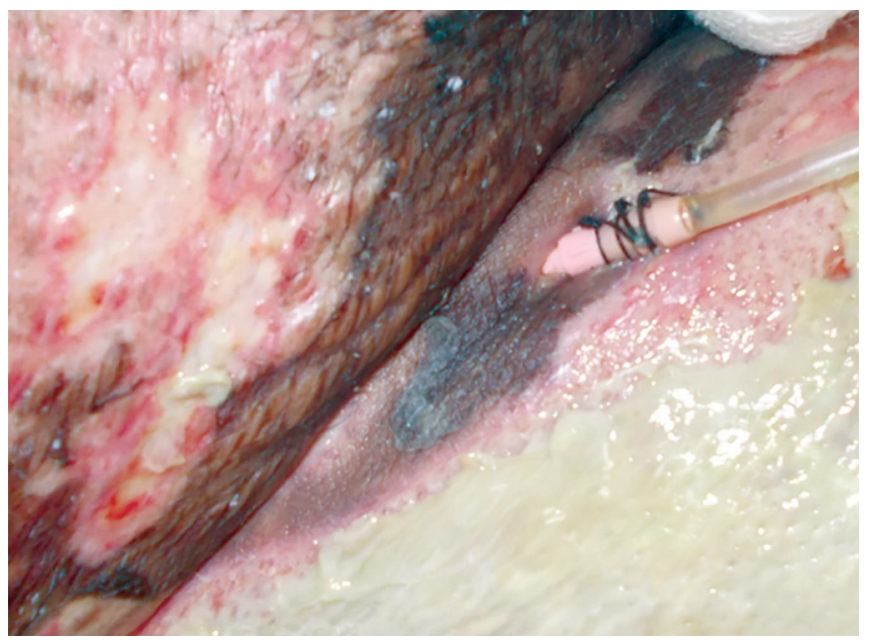

Fig. 2: Cannula inserted very close to the burnt area

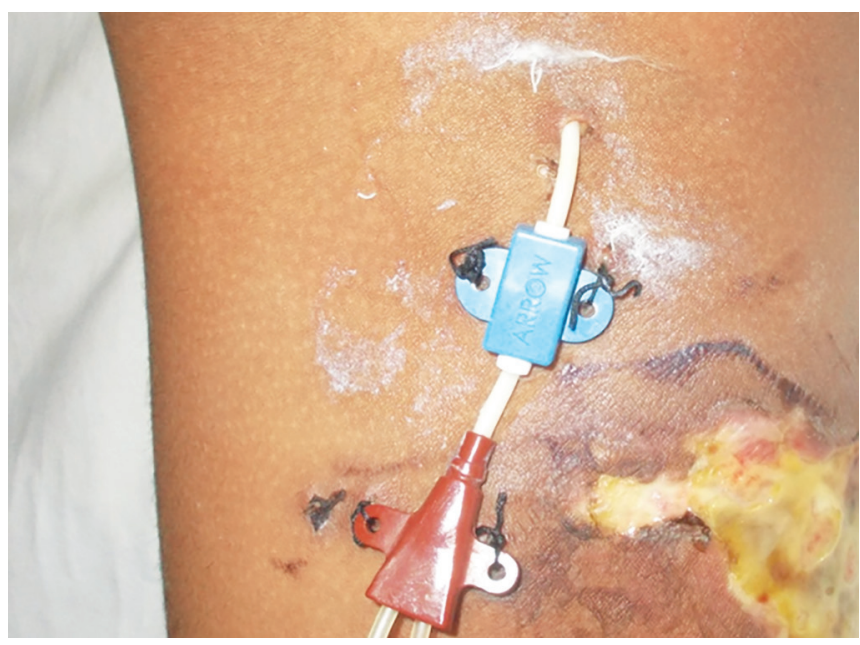

Fig. 4: Exposed cannula inserted at an angle 
DVT compression stockings were applied immediately on being received in the burns unit. If patients could not afford the TED stockings, crepe bandaging from the toes to the groin was done.

Ambulation of the patient was done at the earliest, ideally within 24 hours after resuscitation from the shock, unless the patient was being ventilated for inhalational injuries.

LMWH in the appropriate doses was started in the burns unit immediately on admission. This was done also for patients received from other hospitals but within 72 hours of having sustained burns.

Warfarin was started simultaneously again in the appropriate doses. LMWH was discontinued once the effect of warfarin had set in as determined by the PT/INR. Warfarin was continued for 2-3 weeks depending on the severity of the burn wound.

For cases planned for early excision and skin grafting, Warfarin was discontinued, and patients were shifted to LMWH.

Adequate blood products were kept standby along with protamine. Protamine however never had to be used during the period of the study.

Patients were given prophylactic doses of vitamin K. A very high-calorie high-protein diet (a minimum of about $4000 \mathrm{kcal}$ and $200 \mathrm{~g}$ of proteins) is administered through a nasogastric feeding tube.

\section{Conclusion}

This is a realtively small and short study carried out over a period of 4 years only. It was started in what was a relatively newly established burns unit of a tertiary care teaching hospital. There were no protocols laid down for the management of burns patients as there was no plastic surgeon associated with the unit till 2013.

A relatively high incidence of DVT was noticed in this study. Analysis of the data collected resulted in the initiation of a strictly followed protocol for the prevention, a drug prophyalxis to prevent DVT, and guidelines for treatment of established DVT.

The results of the protocol were remarkable in that there was no incidence of DVT in fresh cases of severe burns admitted to the unit. The only patient in the prospective study was admitted with established DVT as no prophylaxis had been given in the referring hospital.

\section{Clinical Significance}

Though DVT is a well-researched and reported condition, there is very little reliable literature or references available on the incidence or etiopathology of this completely preventable but clinically critical condition in burns patients.

The inordinately high incidence of DVT in a relatively unestablished burns unit was a matter of concern. Hence this study was carried out, and with the completion of the study the incidence of DVT in freshly admitted cases of burns came down to zero.

A well-researched protocol for the prevention of DVT in cases of severe burns has been introduced in the burns unit of the Pravara Rural Hospital with excellent results. It is recommended that a similar protocol may be adopted in various burn units and centres, where such a protocol does not exist. More research and studies may result in India being a pioneering country in the introduction of such a protocol.

\section{References}

1. Ikejiri M, Shindo A, et al. The association of protein STokushima-K196E with a risk of deep vein thrombosis. Int J Hematol 2010;92(2):302-305.

2. National Heart, Lung and Blood institute. http:/www.nhlbi.nih.gov/ health/health-topics/topics/dvt.

3. Herndon DN. Total Burn Care. 5th ed., Elsiever; 2018.

4. Sarabhai S. ed. Principles and Practice of Burns. 1st ed., Jaypee Brothers; 2010.

5. Williams N, Bulstrode C, et al. Bailey and Love's Short Practice of Surgery. 26th ed., Boca Raton: CRC Press; 2013. pp. 262-263.

6. Bhat MS. SRB's Manual of Surgery. 5th ed., Jaypee Brothers Medical P; 2016.

7. Naess IA, Christiansen SC. Incidence and mortality of venous thrombosis: a population based study. J Thromb Hemost 2007;5(4):692-699. DOI: 10.1111/j.1538-7836.2007.02450.x.

8. Kahn SR. The clinical diagnosis of deep vein thrombosis: integrating incidence, risk factors and symptoms and signs. Arch Intern Med 1998;158(21):2315-2323. DOI: 10.1001/archinte.158.21.2315.

9. Tovey C, Wyatt S. Diagnosis, investigation and management of Deep Vein Thrombosis Clinical Review. BMJ 2003;326:1180-1184. DOI: 10.1136/bmj.326.7400.1180.

10. Goldhaber SZ. Pulmonary embolism. Lancet 2004;363:1295. DOI: 10.1016/S0140-6736(04)16004-2.

11. Fecher AM, O'Mara MS, et al. Analysis of deep vein thrombosis in burn patients. Burns 2004 Sep;30(6):591-593. DOI: 10.1016/ j.burns.2003.12.019.

12. Mayon BT, Julian W, et al. Deep vein thrombosis in burns. Burns July 1981;7(6):438-400.

13. Panucci CJ, Osborne NH. Venous thromboembolism in thermally injured patients. Analysis of the national burn repository. J Burn Care Res 2011;32:6-12. DOI: 10.1097/BCR.0b013e318204b2ff.

14. Ahuja RB, Bansal $P$, et al. An analysis of deep vein thrombosis in burn patients: a randomized and controlled study of thromboprophylaxis with Low Molecular Weight Heparin. Burns 2016 Dec;42(8):1693-1698. DOI: 10.1016/j.burns.2016.08.007.

15. Wahl WL, Brandt MM. Potential risk factors for deep evein thrombosis in burn patients. J Burn Care Rehabil 2001;22:128-131. DOI: 10.1097/00004630-200103000-00008.

16. Wahl WL, Barandt MM, et al. Venous thrombus incidence in burn patients - Preliminary results of a prospective study. J Burns Care and Rehab 2002;23(2):206-210. DOI: 10.1097/00004630-200203000-00005.

17. Ann L, Hoballah J, et al. The prevalence of venous thromboembolism of the lower extremity among thermally injured patients determined by duplex sonography. J Trauma 2003;55(6):1162-1167.

18. Faucher CD, Lohlon KM. Practice guidelines for deep vein thrombosis in burns. J Burn Care Res 2007 Sep-Oct;285:661-663. DOI: 10.1097/ BCR.0B013E318148C887.

19. Lipe B, Ornstein DL. Deficiencies of natural anticoagulants, Protein C, protein S, and antithrombin. Circulation 2011;124(14):e365-e368. DOI: 10.1161/CIRCULATIONAHA.111.044412.

20. Wells PS, Owen C. Does this patient have deep vein thrombosis? JAMA 2006 Jan 11;295(2):199-207. DOI: 10.1001/jama.295.2.199, (Discussion of Wells DVT score).

21. Snow V, et al. Management of venous thromboembolism: A clinical practice guideline from the American College of Physicians and the American Academy of Family physicians. Ann Intern Med 2007;146(3):204-210. DOI: 10.7326/0003-4819-146-3-200702060-00149.

22. Lederle FA, et al. Venous thromboembolism prophylaxis in hospitalized patients: A background review for an American College of Physicians clinical practice guideline. Ann Intern Med 2011;155(9):602-615. DOI: 10.7326/0003-4819-155-9-201111010-00008.

23. Bates SM, Jaeschke R, et al. Diagnosis of DVT. Chest 2012 Feb;141(2): e351S-418S. DOI: 10.1378/chest.11-2299. 